\title{
Evaluation of fatty acids in groomed fingerprint by gas chromatographic analysis using various extraction solvents and treatment methods
}

\author{
Youngmin Kim ${ }^{1,8}$, Won-sil Choi ${ }^{2}$, Eun Ji Choi ${ }^{2}$, Byoungjun Jeon ${ }^{3,8}$, Jungah Kim ${ }^{4,8}$, Gee Ho Park ${ }^{1,8}$, Yan Huang ${ }^{1,8}$,

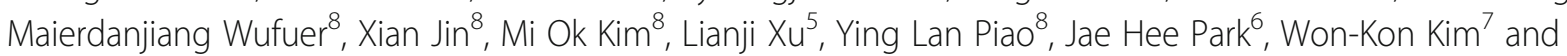 \\ Tae Hyun Choi ${ }^{8^{*}}$
}

\begin{abstract}
Extremely small amounts of fatty acids detected in latent fingerprints are important for studying fingerprint visualization and age determination through changes in composition over time. However, methods for efficiently extracting or recovering fatty acids from fingerprints have not been extensively studied. If accurate and stable quantitative estimations are established, age estimates will be possible through a better understanding of the fatty acid composition. The extraction solvent and treatment method are essential factors for achieving a reliable analysis of fatty acids. There have been few previous studies that efficiently compared fatty acids. In this study, fatty acids from sebaceous fingerprint residues were quantified with various extraction solvents and treatment methods and were evaluated with gas chromatography flame ionization detection (GC-FID). All data were analyzed using a statistical method.
\end{abstract}

Keywords: Latent fingerprint, Gas chromatography flame ionization detector, Fatty acids, Methylation, Extraction solvent

\section{Introduction}

Intrinsic components detected in latent fingerprints have already been identified through numerous forensic studies as an important index for the development of fingerprints (Gird et al. 2012; Bailey et al. 2016; Ramotowski et al. 2001; Rohatgi and Kapoor 2016; Bohanan 1998; Asano et al. 2002; Croxton et al. 2010; Stewart et al. 1989; Antoine et al. 2010; Michalski et al. 2013a; Archer et al. 2005; Cadd et al. 2015a; Cadd et al. 2015b). These studies showed that the components of a fingerprint are 95-99\% water and organic (protein, amino acids, urea, and lipids) and inorganic (sodium, potassium, chloride, and phosphate) compounds secreted from eccrine, apocrine, and sebaceous glands. Among these components, lipids, included glycerides (33\%), fatty acids (30\%), wax esters (22\%), cholesterol esters (2\%), cholesterol (2\%),

\footnotetext{
* Correspondence: psthchoi@snu.ac.kr

${ }^{8}$ Department of Plastic and Reconstructive Surgery, Institute of

Human-Environment Interface Biology, College of Medicine, Seoul National

University, 101 Daehak-ro, Jongno-gu, Seoul, South Korea

Full list of author information is available at the end of the article
}

and squalene $(10 \%)$, make up a large portion of the latent fingerprint in organic compounds (Gird et al. 2012; Bailey et al. 2016; Ramotowski et al. 2001). These compounds originate from the sebum secreted by the sebaceous glands and are very useful ingredients to visualize fingerprints (Rohatgi and Kapoor 2016). Fatty acids are used as a particular target ingredient, which is important for aging studies due to its oxidation over time and the fact that it can be used to identify donor gender, age, eating habits, and race (Bohanan 1998; Asano et al. 2002; Croxton et al. 2010; Stewart et al. 1989; Antoine et al. 2010; Michalski et al. 2013a; Archer et al. 2005; Cadd et al. 2015a). A better understanding of the composition changes over time is required to improve detection and visualization of the latent fingerprint and can also be useful in providing clues to criminal investigations (Cadd et al. 2015b; Girod et al. 2016).

In previous studies, analysis of fatty acids in fingerprints varied depending on the researcher, including sampling protocol, type of fingerprint deposition substrate, extraction solvent, treatment methods, and 
derivatization. Furthermore, some methods showed unavoidable limitations due to their complexity, not using actual fingerprints, low recovery ratio, high loss, uneven samples, time-consuming methods, need for toxic solvents, and use of expensive materials (Bohanan 1998; Asano et al. 2002; Cadd et al. 2015a; Mong et al. 1999; Weyermann et al. 2011; Mink et al. 2013; Croxton et al. 2006; Koenig et al. 2011; Girod and Weyermann 2014).

Mong et al. (1999) used micro filter paper extracted in a serological pipette with 1:1 hexane:chloroform $(1 \mathrm{~mL})$ followed by acetone $(1 \mathrm{~mL})$. The sample was analyzed by gas chromatography mass spectrometry (GC-MS) after being derivatized with diazomethane. This method showed 19 components of fatty acids with cholesterol and squalene in young and adult donors.

Asano et al. (2002) used chloroform $\left(\mathrm{CHCl}_{3}\right)$ as an extraction solvent and performed analysis by GC-MS without any derivatization. This method showed 6 components of fatty acids in 10 males and 10 females with fatty acid esters and cholesterol. However, one of the drawbacks was that samples deposited on glass beads were not similar to actual fingerprints.

Archer et al. (2005) employed dichloromethane $\left(\mathrm{CH}_{2} \mathrm{Cl}_{2}\right)$ with chlorononane $(\mathrm{CN})$ in hexane and analyzed the samples using GC-MS after they were derivatized with $\mathrm{N}$-methyl- $N$-(trimethylsilyl)-tri-fluoroacetamide (MSTFA). In this method, fingerprints were deposited on small pieces of glass fiber filter paper and were collected into vials. The result showed 5 components of fatty acids from 5 males. However, one of the limitations was that considerable variation was observed due to uneven distribution of fingerprints, uneven pressure application, and uneven splitting of fingerprint sample.

Seth et al. (2013a) used a glass microfiber filter to remove fingerprints from glass slides soaked in 2:1 chloroform/methanol and analyzed by GC-MS. This method showed 12 fatty acids in 37 donors. However, the average percentage recovery from the seven trials was low, $37.6 \%$.

Table 1 Characteristics of the fingerprint donors

\begin{tabular}{lllll}
\hline Donor name & Gender & Age & Smoke & Cosmetics \\
\hline A & Male & 37 & No & Facial lotio \\
B & Male & 35 & No & Facial lotio \\
C & Female & 26 & No & Face cream \\
D & Female & 27 & No & Face cream \\
E & Male & 28 & No & Facial lotio \\
F & Male & 27 & Yes & Facial lotio \\
G & Female & 36 & No & Face cream \\
H & Male & 38 & No & Face cream \\
I & Female & 26 & No & Face cream \\
J & Male & 50 & Yes & Facial lotio \\
\hline
\end{tabular}

The reason for this is that the evaporation of extraction solvent caused high loss and low recovery.

Cadd et al. (2015a) investigated the differences between reported extraction and separation methods. To determine the extraction efficiency, they used a glass coverslip and 7 solvent methods for preliminary extraction. The result showed that fatty acids were most successfully extracted from samples deposited on the glass coverslip with $\mathrm{MeOH} / \mathrm{TMSCL}$. However, the calculated extraction efficiency was determined for the three fatty acids $(45 \%$ for dodecanoic acid, $69 \%$ for stearic acid, $69 \%$ for nonanoic acid). The most important factors were the solvent, which must completely dissolve the fatty acids, and a treatment method that does not result in maximum loss.

Thus, to efficiently recover fatty acids, an appropriate extraction solvent and an optimal treatment method must be selected depending on the characteristics of the substrate on which the fingerprint is transferred (Michalski et al. 2013b). So far, there has not been much research on the recovery ratio of components in fingerprints.

In this study, methods for efficiently recovering fatty acids by using five extraction solvents and four treatment methods were evaluated. Qualitative and quantitative results were analyzed using GC-FID after methylation process. In general, the analysis of fatty acids in fingerprint was performed by GC-MS, which has the ability to confirm the identity of fatty acids based on spectral information and retention time. However, GC-FID was chosen in this research. The reason is that it has a quantitative performance similar to GC-MS as well as lots of advantages including cheaper, easier to operate, and time saving (Dodds et al. 2005; Young et al. 2012). Most fatty acids analyses by $\mathrm{GC}$ require derivatization due to the high boiling points of fatty acids (Laakso et al. 2002; Godswill et al. 2014).

According to the International Fingerprint Research Group (IFRG) guideline, this study is recommended to be performed in natural fingerprints. However, since the amount of fatty acid detected in natural fingerprints is too small, it is difficult to perform recovery and quantification (Guidelines for the assessment of fingermark detection techniques, International Fingerprint Research Group (IFGR) 2014). So, we analyzed the fingerprints

Table 2 Five extraction solvents

\begin{tabular}{ll}
\hline Extraction solvents & \\
\hline 1 & Chloroform \\
2 & Chloroform:methanol (3:1) \\
3 & Acetone:chloroform:hexane \\
& $(1: 1: 1)$ \\
4 & Hexane:isopropanol (3:2) \\
5 & Chloroform:ethanol (1:1) \\
\hline
\end{tabular}


Table 3 Sample classification method using different extraction solvents

\begin{tabular}{llllll}
\hline Donor name & Thumb & Index & Middle & Ring & Little \\
\hline A & 1 & 2 & 3 & 4 & 5 \\
B & 5 & 1 & 2 & 3 & 4 \\
C & 4 & 5 & 1 & 2 & 3 \\
D & 3 & 4 & 5 & 1 & 2 \\
E & 2 & 3 & 4 & 5 & 1 \\
F & 1 & 2 & 3 & 4 & 5 \\
G & 5 & 1 & 2 & 3 & 4 \\
H & 4 & 5 & 1 & 2 & 3 \\
I & 3 & 4 & 5 & 1 & 2 \\
J & 2 & 3 & 4 & 5 & 1 \\
\hline n= 10, total sample $=50$. Treatment method & cotton swab &
\end{tabular}

$n=10$, total sample $=50$. Treatment method $=$ cotton swab

after touching face to get enough amount to analyze. First, evaluation of the recovery ratio by the five extraction solvents was carried out onto cover glass deposited fingerprint residues with undecanoic acid (C11) and they were then collected using a cotton swab. The cotton swab method is commonly employed for DNA and protein collection (Zhang et al. 2015; Bobev 1995; Steadman et al. 2015). Secondly, a study of the recovery ratio of four treatment methods was conducted using a solvent that had a high recovery efficiency, as determined in the previous experiment. Third, the calculated recovery ratio was compared with the amount recovered from the groomed fingerprint.

\section{Materials and methods Materials}

Capric acid (C10, $\geq 98 \%$ ), undecanoic acid (C11, $\geq 98 \%$ ), lauric acid (C12, $\geq 98 \%)$, myristic acid (C14, $\geq 99.5 \%$ ), pentadecaonic acid (C15, $\geq 99 \%)$, palmitic acid $(\mathrm{C} 16, \geq$ 99\%), stearic acid (C18, $\geq 98.5 \%)$, oleic acid (C18:1n9c, $\geq$ 99\%), arachidic acid (C20, $\geq 99 \%$ ), behenic acid (C22, $\geq$ 99\%), and lignoceric acid (C24, $\geq 99 \%$ ) were obtained from Sigma-Aldrich. Ethyl alcohol (anhydrous, $\geq 99.9 \%$, methanol ( $\geq 99.8 \%$ ), hexane ( $\geq 99.9 \%)$, 2-propanol (isopropanol $\geq 99.5 \%$ ), acetone, heptane, and chloroform were of analytical-reagent or GC grade and were obtained from Fisher Scientific UK Limited and Sigma-

Table 4 Four treatment methods

\begin{tabular}{ll}
\hline Treatment methods & \\
\hline 1 & Rotator (shaking) \\
2 & Cotton swab (swabbing) \\
3 & Pipette (handling) \\
4 & Tape (lifting) \\
\hline
\end{tabular}

Aldrich. Benzene ( $\geq 99.8 \%)$, dimethoxylpropane $(\geq$ $99.8 \%$ ), and sulfuric acid ( $\geq 99 \%$ ) were volumetric analysis grade and were also obtained from Sigma-Aldrich. Fatty acid standards (Supelco 37 Component FAME Mix) were obtained from Supelco (USA). Deckgläser, microscope cover glasses, $24 \times 24 \mathrm{~mm}$ was purchased from Paul Marienfeld GmbH \& Co.KG (Lauda-Königshofen, Germany) and were utilized for sample collection. Scotch tape $(18 \mathrm{~mm} \times 2 \mathrm{~m})$ and cotton swabs were obtained from 3M (Seoul, Korea) and were used for sample collection. A 4-mL amber glass vial with a Teflon-lined cap was purchased from Wheaton ${ }^{\circ}$ and was utilized for sample collection.

\section{Methods}

\section{Sample preparation}

Six male and four female donors between 26 and 50 years old were selected for this study. All donors were Asian and were omnivores who had no diagnosed metabolic disease (Table 1). Two donors were smokers and there were no reported illnesses. Informed consent from all donors (IRB No. 1610-009-795) was obtained, and they were anonymized with an alphanumeric code. First, the cover glasses were cleaned using acetone and dried at room temperature. Then, the fingerprint sample was obtained. The donors were asked to follow the three steps listed as follows.

1. The donors were requested to washed their hands with soap and wiped them thoroughly with a tissue (KIMTECH, Wiper, Yuhan-Kimberly, Korea) soaked in ethanol (70\%).

2. The donors gently rubbed all their fingers across the cheek, nose, and forehead regions of their face for approximately $5 \mathrm{~s}$ and then rubbed them against each other to evenly distribute the lipid ingredients. This activity was intended to distribute the sebaceous secretions of the face onto the fingertips.

Table 5 Sample classification method using different treatment methods

\begin{tabular}{llllll}
\hline Donor name & Thumb & Index & Middle & Ring & Little \\
\hline A & 1 & 2 & 3 & 4 & 1 \\
B & 2 & 1 & 2 & 3 & 4 \\
C & 4 & 3 & 1 & 2 & 3 \\
D & 3 & 4 & 4 & 1 & 2 \\
E & 1 & 2 & 3 & 4 & 1 \\
F & 2 & 1 & 2 & 3 & 4 \\
G & 4 & 3 & 1 & 2 & 3 \\
H & 3 & 4 & 4 & 1 & 2 \\
\hline$n=10$, total sample $=$ 40. Extraction solvent $=$ chloroform:ethanol $(1: 1, \mathrm{v} / \mathrm{v})$
\end{tabular}


Table 6 List of targeted fatty acids in GC-FID analysis

\begin{tabular}{|c|c|c|c|}
\hline 1 & Butyric acid (C4) & 3.383 & 2.1 \\
\hline 2 & Caproic acid (C6) & 4.575 & 2.78 \\
\hline 3 & Caprylic (C8) & 5.825 & 3.3 \\
\hline 4 & Capric (C10) & 7.243 & 3.66 \\
\hline 5 & Undecanoic (C11) & 8.085 & 1.88 \\
\hline 6 & Lauric (C12) & 8.939 & 3.93 \\
\hline 7 & Tridecanoic (C13) & 9.889 & 1.98 \\
\hline 8 & Myrisric (C14) & 10.897 & 4.11 \\
\hline 9 & Myristoleic (C14:1) & 11.51 & 2.02 \\
\hline 10 & Pentadecanoic (C15) & 12.266 & 2.09 \\
\hline 11 & cis-10-Pentadecanoic (C15:1) & 12.894 & 1.97 \\
\hline 12 & Palmitic (C16) & 13.764 & 6.38 \\
\hline 13 & Palmitoleic (C16:1) & 14.296 & 2.1 \\
\hline 14 & Heptadecanoic (C17) & 15.489 & 1.82 \\
\hline 15 & cis-10-Heptadecenoic (C17:1) & 16.111 & 2.09 \\
\hline 16 & Stearic (C18) & 17.45 & 4.42 \\
\hline 17 & Elaidic (C18:1n9t) & 17.773 & 2.15 \\
\hline 18 & Oleic (C18:1n9c) & 17.991 & 4.37 \\
\hline 19 & Linolelaidic (C18:2n6t) & 18.527 & 0.96 \\
\hline 20 & Linoleic (C18:2n6c) & 19.089 & 2.1 \\
\hline 21 & Y-Linolenic (C18:3n6) & 19.76 & 2.06 \\
\hline 22 & a-Linolenic (C18:3n3) & 20.524 & 2.2 \\
\hline 23 & Arachidic (C20) & 21.946 & 4.42 \\
\hline 24 & cis-11-Eicosenoic (C20:1n9) & 22.588 & 2.22 \\
\hline 25 & cis-11,14-Eicosadienoic (C20:2) & 23.891 & 2.13 \\
\hline 26 & Heneicosanoic (C21) & 24.408 & 2.22 \\
\hline 27 & cis-8,11,14-Eicosatrienoic (C20:3n6) & 24.635 & 1.69 \\
\hline 28 & Arachidonic (C20:4n6) & 25.097 & 1.92 \\
\hline 29 & cis-11,14,17-Eiosatrienioic (C20:3n3) & 25.516 & 1.95 \\
\hline 30 & Behenic (C22) & 26.76 & 1.88 \\
\hline 31 & Eicosapentaenoic (C20:5n3) EPA & 26.94 & 4.52 \\
\hline 32 & Erucic (C22:1n9) & 27.664 & 2.19 \\
\hline 33 & Docosadienoic (C22:2) & 29.077 & 2.12 \\
\hline 34 & Tricosanoic (C23) & 29.509 & 2.29 \\
\hline 35 & Lignoceric (C24) & 32.099 & 4.7 \\
\hline 36 & Docosahexaenoic (C22:6n3) DHA & 32.745 & 1.92 \\
\hline \multirow[t]{2}{*}{37} & Nervonic (C24:1n9) & 32.93 & 2.27 \\
\hline & Total & & 100 \\
\hline
\end{tabular}

3. Fingerprints were deposited on each microscope cover glass $(24 \times 24 \mathrm{~mm}$ diameter $)$, which is a nonporous flat surface. From each donor, 10 fingerprint samples were collected. The time of deposition was maintained at approximately $5 \mathrm{~s}$ and the same touch angle. The fingerprint samples were stored in the dark at room temperature until extraction. The extraction was conducted the following day.

\section{Sampling: choice of extraction solvents}

Prior to the extraction process, $20 \mu \mathrm{L}$ of solution of C11 fatty acid ( $2 \mathrm{mg} / \mathrm{mL}$ hexane) as a recovery standard was dropped onto all fingerprint samples, and the solvent was removed by standing in the air for $10 \mathrm{~s}$. Here, the treatment method was executed using a cotton swab. The cotton swab was completely immersed in a 4-mL amber vial with $2 \mathrm{~mL}$ of the chosen solvent (Table 2), and then, the fingerprint on the glass surface was swabbed twice. The cotton swab end was cut with a cleaned scalpel and placed into the amber glass vial. The amber vial was immediately sealed with a Teflon-lined cap and sonicated in an ultrasonic bath to extract fatty acids from the cotton swab for $10 \mathrm{~min}$. After $10 \mathrm{~min}$, the cotton swab in the vial was removed using a cleaned tweezers, and then, the amber vial without the cap was evaporated to dryness using a freeze dryer (HyperVAC, BSM, Korea) for $1 \mathrm{~h}$ at $2000 \mathrm{rpm}$ and $28^{\circ} \mathrm{C}$ under vacuum. Dried amber vials containing extract were sealed with a cap and stored at $-80^{\circ} \mathrm{C}$ until the next derivatization process. A cover glass without deposited fingerprint residue was also processed to serve as blanks. Sample classification methods (Table 3) showed that five fingers per donor were deposited on the cover glass, and a different extraction solvent was applied to each fingerprint with sequential rotation for every donor.

\section{Sampling: choice of extraction treatment}

Four types of treatment methods were used in this study (Table 4). Chloroform:ethanol (1:1, v/v) was selected as the extraction solvent. The first type of method used a rotator (shaking). In this process, the cover glass deposited fingerprint was cut into small pieces using a scalpel and was placed in a 4-mL amber glass vial. A 2-mL extraction solvent was added to the amber glass vial and was shaken using a rotator (200 cycles/min). After 10 min, small pieces of glass were removed. The second type of method employed cotton swab (swabbing). The cotton swab was placed in the amber vial containing the chosen solvent and was completely immersed. After the fingerprint in the sample was thoroughly wiped twice, the cotton swab end was cut off with a cleaned scalpel, placed into the amber vial, and sonicated for $10 \mathrm{~min}$. After $10 \mathrm{~min}$, the cotton swab in the vial was removed using a tweezers. The third type of method used a pipette (handling). Specifically, $200 \mu \mathrm{l}$ of extraction solvent was dropped on the fingerprint sample, and the surface was rubbed with a carefully handled pipette. Extra extraction solvent on the surface was repeatedly collected in the amber vial (five times). The last type of method utilized tape (lifting). The fingerprint samples on the 


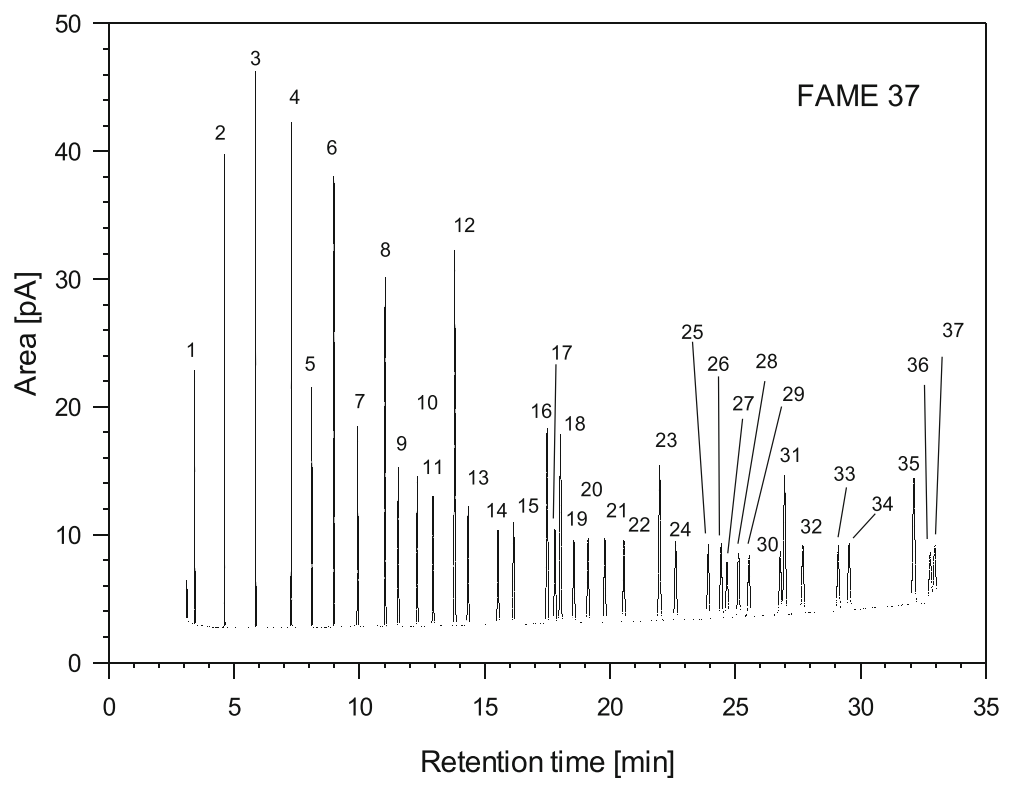

Fig. 1 GC-FID chromatogram of 37 fatty acid methyl esters

cover glass surface were collected with scotch tape (18 $\mathrm{mm} \times 2 \mathrm{~m}$ ) five times, put into an amber vial containing 2 - $\mathrm{mL}$ extraction solvent, and sonicated for $10 \mathrm{~min}$. The drying process and preparation of blank samples were carried out in the same manner as described earlier. Sample classification methods were also conducted using the same process as described earlier.

\section{Sampling: preparation of fatty acid methyl ester (FAME)} For methylation of fatty acid for GC analysis, dried amber vial containing extract was filled with $1 \mathrm{~mL}$ of the mixture MeOH:benzene:dimethoxypropane: $\mathrm{H}_{2} \mathrm{SO}_{4}=$ 39:20:5:2 (v/v/v/v), $0.5 \mathrm{~mL}$ heptane, and $20 \mu \mathrm{L}$ of an internal standard solution $\mathrm{C} 15(5.0 \mathrm{mg} / \mathrm{mL})$; the mixture was shaken for $5 \mathrm{~s}$ and heated on a hot plate at $85^{\circ} \mathrm{C}$ for $2 \mathrm{~h}$ with shaking at $1 \mathrm{~h}$ interval (Garcés \& Mancha, 1993). When the reaction was completed, vial was cooled to room temperature over approximately $30 \mathrm{~min}$.

Table 7 Fatty acid compounds detected in latent fingerprint residue from donor $\mathrm{A}$ by $\mathrm{GC}$ analysis

\begin{tabular}{llll}
\hline $\begin{array}{l}\text { Target compound } \\
\text { (bond) }\end{array}$ & $\begin{array}{l}\text { Retention } \\
\text { time (min) }\end{array}$ & $\begin{array}{l}\text { Relative } \\
\text { abundance (\%) }\end{array}$ \\
\hline 12 & Palmitic acid (C16) & 13.78 & 41.96 \\
16 & Stearic acid (C18) & 17.46 & 23.85 \\
18 & Oleic acid (C18:1n9c) & 18 & 6.73 \\
23 & Arachidic acid (C20) & 21.96 & 5.07 \\
30 & Behenic acid (C22) & 26.76 & 8.69 \\
32 & Erucic acid (C22:1n9) & 27.67 & 4.66 \\
\hline
\end{tabular}

Then, the upper layer (heptane layer) containing the FAMEs and the internal standard was transferred into a 2-ml auto-sampler glass and stored at $-80^{\circ} \mathrm{C}$ until GCFID analysis (Table 5).

\section{GC-FID analysis}

Methylated fatty acid analysis was quantitatively performed on an Agilent 7890A (Agilent, USA) gas chromatography equipped with a flame ionization detector (GC-FID), using a J\&W DB-23 capillary column $(60 \mathrm{~m} \times 0.25 \mathrm{~mm} \times 0.25$ $\mu \mathrm{m}$, Agilent, USA). The injection and detector temperatures were set to $250{ }^{\circ} \mathrm{C}$ and $280{ }^{\circ} \mathrm{C}$, respectively. Helium was used as a carrier gas with a flow rate of $1.0 \mathrm{ml} / \mathrm{min}$. The split ratio was 10:1 in duplicate, and $1 \mu \mathrm{L}$ of the sample solution was injected. The column was held at $50{ }^{\circ} \mathrm{C}$ for $1 \mathrm{~min}$ and then ramped to $130{ }^{\circ} \mathrm{C}$ at $15^{\circ} \mathrm{C} / \mathrm{min}$. It was then ramped at $8{ }^{\circ} \mathrm{C} / \mathrm{min}$ to $170{ }^{\circ} \mathrm{C}$, ramped at $2{ }^{\circ} \mathrm{C} / \mathrm{min}$ to $215^{\circ} \mathrm{C}$, and held for $10 \mathrm{~min}$. Finally, it was ramped at $5^{\circ} \mathrm{C} /$ min to $250^{\circ} \mathrm{C}$ and held for $5 \mathrm{~min}$.

FAME was identified by comparison of their retention times to those of authentic standards analyzed under the same conditions as shown in Table 6 and Fig. 1. The compositions of fatty acid correlated FAMEs in sample were calculated on the basis of the percentage of total fatty acid and according to the peak attained from analyzing. The identification of a peak was made from its retention time (Dodds et al. 2005).

\section{Statistical analysis}

All statistical analyses were performed by GraphPad Prism statistical software (version 5.0 for windows, GraphPad 


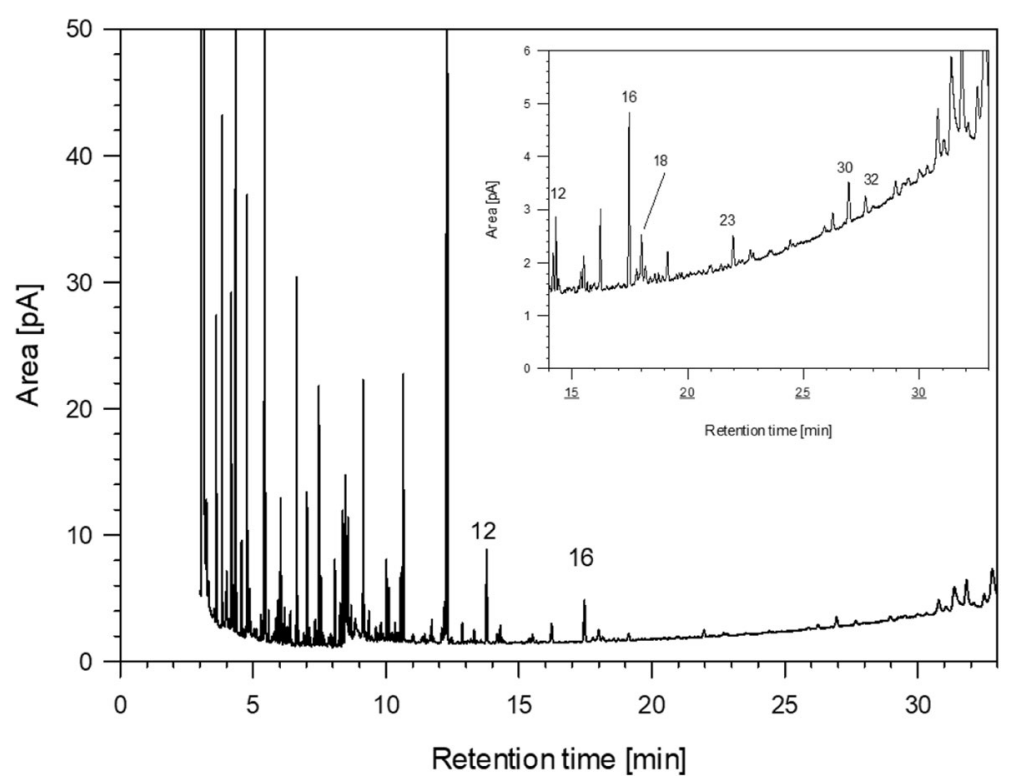

Fig. 2 A GC-FID chromatogram of fingerprint residue from donor A

software, La Jolla, CA, USA). All data from extraction solvents and collection methods were conducted using a one-way ANOVA for comparing groups, and Tukey's multiple comparison tests was used to determine the statistical difference. All graph bars are presented as the

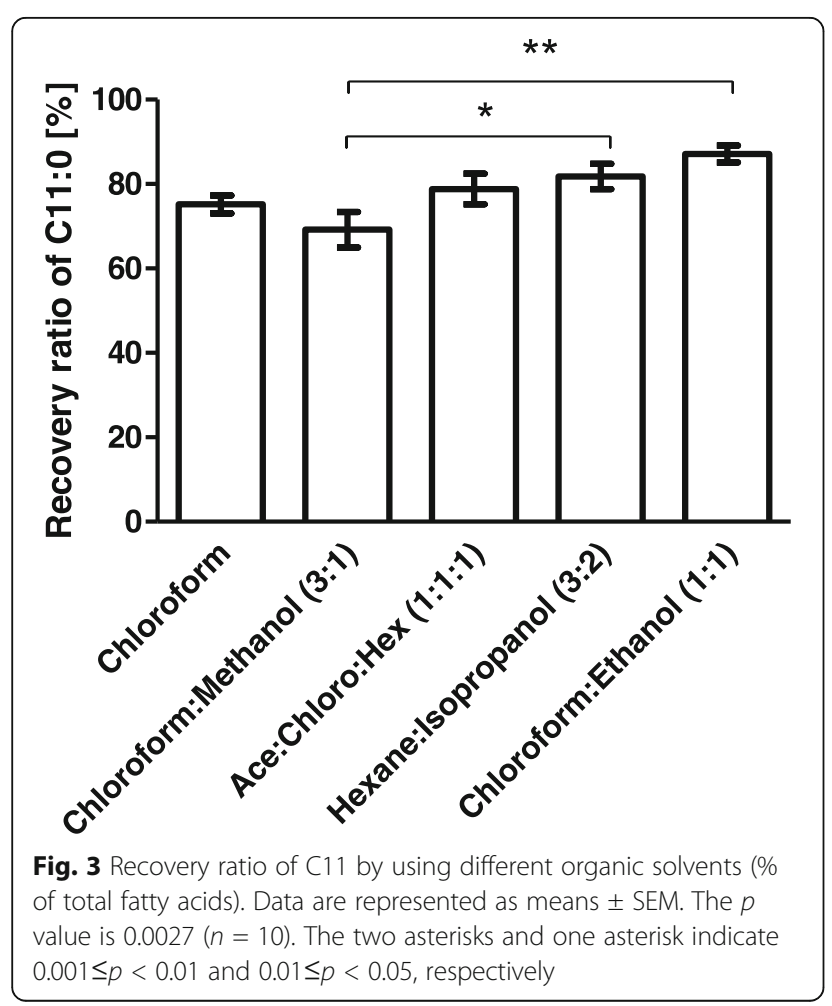

means \pm SEM (standard error of the mean). The degree of statistical difference is indicated by the $p$ value and by ${ }^{* * * * *}$, $* * * * *$, and * for $p \leq 0.0001,0.0001 \leq p<0.001,0.001 \leq p$ $<0.01$, and $0.01 \leq p<0.05$, respectively. "No SD" means no significant difference.

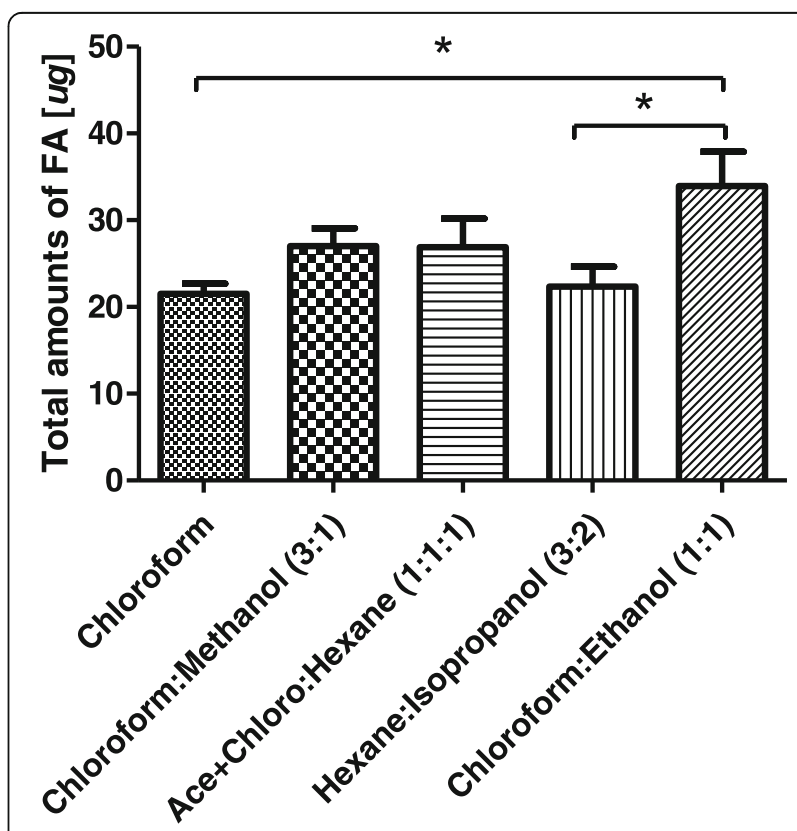

Fig. 4 Total quantity of fatty acids by using different organic solvents ( $\mu \mathrm{g}$ of total fatty acids). Data are represented as means \pm SEM. The $p$ value is 0.011 ( $n=10$, all donors). The one asterisk indicates $0.01 \leq p<0.05$ 

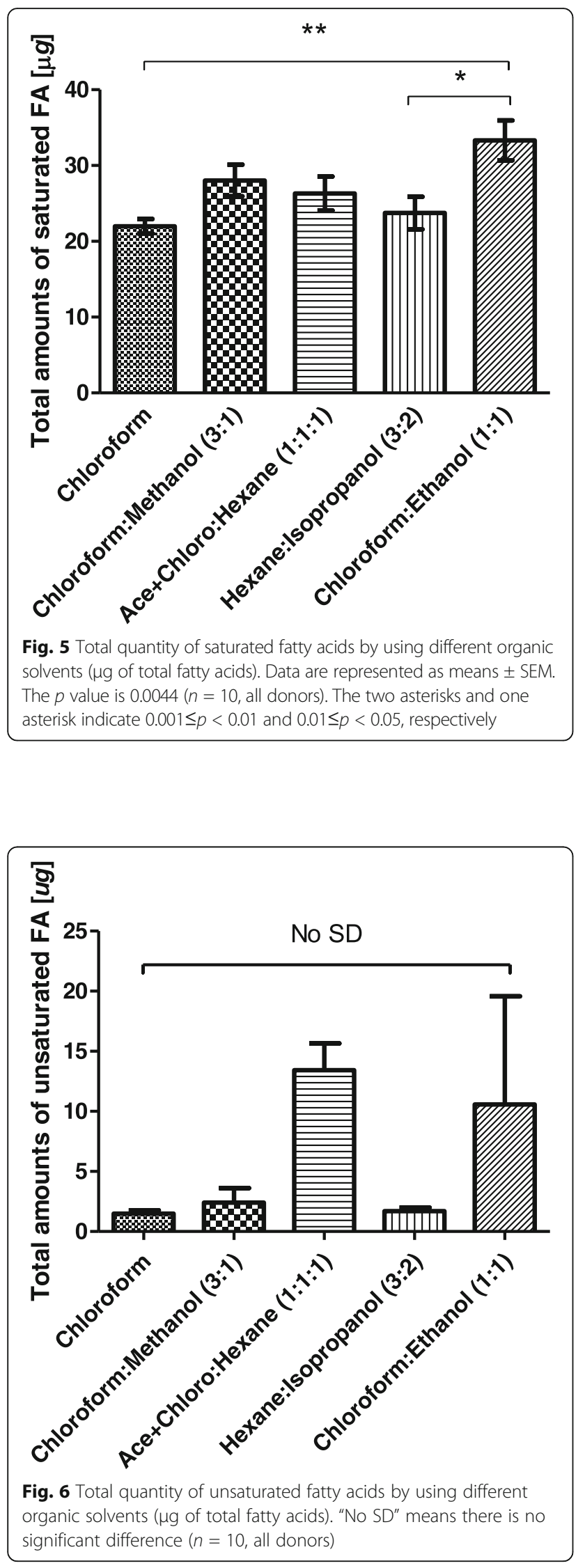

\section{Results}

Determination of fatty acids

Groomed fingerprint samples were successfully collected from 10 donors and were extracted from the cover glass using each of the five extraction solvents and four treatment methods. GC analysis of FAMEs was carried out using two internal standards. Specifically, undecanoic acid (C11:0) (for calculating \% recovery) was added prior to the transesterification and pentadecanoic acid (C15:0) was also added directly before the analysis to determine concentration of samples. Analysis of fatty acids from fingerprints generally involves three steps: extraction of fatty acids from fingerprints on the cover glass, conversion of the extracted fatty acids methyl esters (FAME), and analysis of the FAME using gas chromatography with a flame ionization detector (GC-FID) for fatty acid identification. Table 7 and Fig. 2 show fatty acid compounds detected in groomed fingerprint residue and a GC-FID chromatogram from donor A.

\section{Fatty acid recovery ratio and quantification by five extraction solvents}

To determine the accuracy of the method, standard solutions were employed. The accuracy was expressed as a percent recovery ratio determined by calculation of the mean recoveries $\pm \mathrm{SD}$ of several levels of concentrations. The recovery percentage for each solvent was calculated from Eq. (1).

$$
R j(\%)=\frac{\bar{x}_{\mathrm{obs}_{j}}}{\bar{x}_{\mathrm{ref}_{j}}} \times 100
$$

Here, $R_{j}$ is the recovery of sample $\mathrm{j}, \bar{x}_{\text {obs }}$ is the average concentration of compound $j$ determined from GC analysis and $\bar{x}_{\text {ref }}$ is the actual concentration of $j$ in the standard samples (Godswill et al. 2014).

The results of fatty acid recovery ratios using five organic solvents were obtained and showed variation among the solvent systems. The highest recovery ratio for C11 was obtained with chloroform:ethanol $(1: 1, \mathrm{v} / \mathrm{v}$, $87 \%)$, followed by hexane:isopropanol $(3: 2, \mathrm{v} / \mathrm{v}, 82 \%)$, acetone:chloroform:hexane (1:1:1, v/v/v, 79\%), chloroform (75\%), and chloroform:methanol (3:1, v/v, 69\%), (Fig. 3). All recovery trials were conducted in 10 repetitions. Recoveries (100\%) are calculated using standards that do not require extraction or treatment for comparison with treated samples. A significant difference was exhibited in two comparisons: chloroform:methanol (3: 1) vs. hexane:isopropanol (3:2) and chloroform:methanol (3:1) vs. chloroform:ethanol (1:1). The largest amount of fatty acids collected from donors was obtained in chloroform:ethanol (1:1) groups. A significant difference was observed for the chloroform vs. chloroform:ethanol (1:1) and hexane: isopropanol (3:2) vs. chloroform:ethanol (1: 


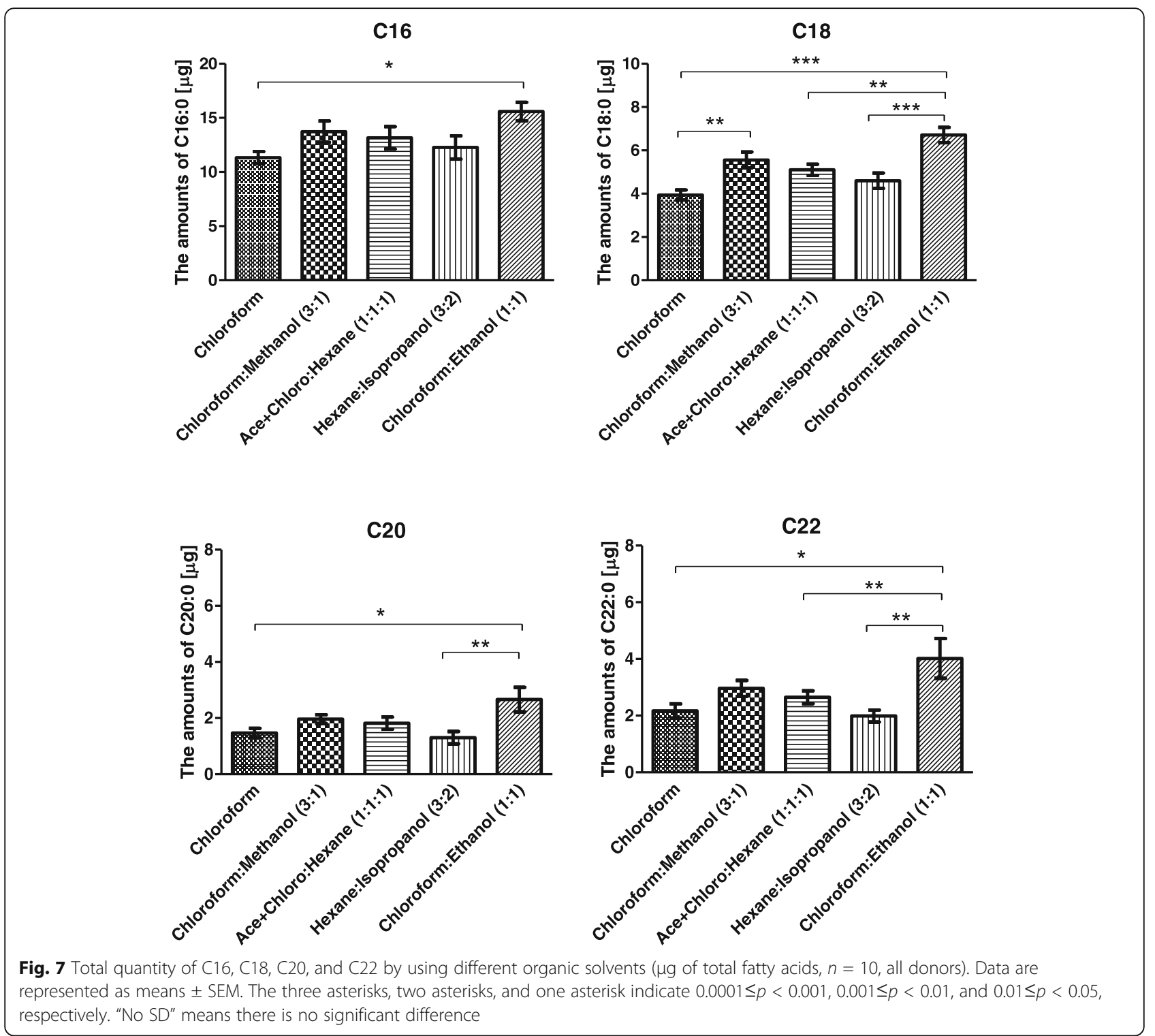

1) group (Fig. 4). From the 10 donors, eight fatty acids were obtained with total amounts of 16.1-62.8 $\mu \mathrm{g}$ per print. The detected saturated fatty acids were C14, C16, $\mathrm{C} 18, \mathrm{C} 20$, and $\mathrm{C} 22$, and unsaturated fatty acids were C18:2n6c, C18:3n3, and C22:1n9. Saturated fatty acids (C16, C18, C20, and C22) were common factors in all fingerprint deposits. However, a few saturated fatty acids (C14) and all unsaturated fatty acids showed donor dependence. Despite the variability in saturated and unsaturated fatty acids, the saturated fatty acids from donors showed significant differences between chloroform vs. chloroform:ethanol (1:1) and hexane:isopropanol (3:2) vs. chloroform:ethanol (1:1) (Fig. 5). No significant differences were found for unsaturated fatty acids. However, the largest amount of unsaturated fatty acids was obtained with chloroform:ethanol (1:1) (Fig. 6). Among the saturated fatty acids detected in all donors, C16 $(8.8-22 \mu \mathrm{g})$ was obtained in the highest relative amounts, followed by C18 (3.1-9.4 $\mu \mathrm{g})$, C22 (1.4-9.4 $\mu \mathrm{g})$, and $\mathrm{C} 20(0.8-5.8 \mu \mathrm{g})$. The quantity recovered from extraction solvents showed that chloroform:ethanol (1:1) best extracted the remaining saturated fatty acids at statistically significant levels (Fig. 7). GC-FID chromatograms of fingerprint residue from donor $\mathrm{E}$ showed variations in the quantity of recovered fatty acids based on extraction solvent (Fig. 8).

\section{Fatty acid recovery ratio by extraction treatment}

Fatty acids were most successfully extracted from deposited glass surface using chloroform:ethanol (1:1). The 


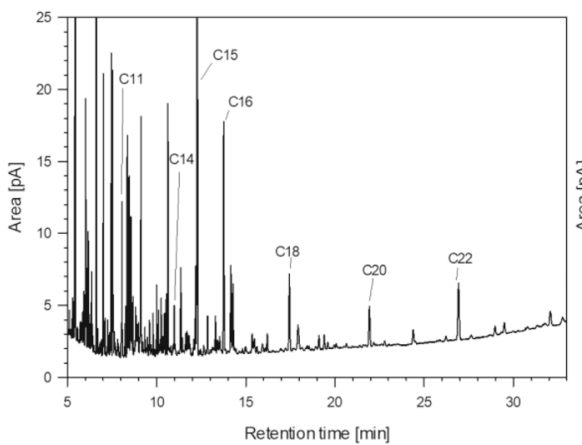

(i)

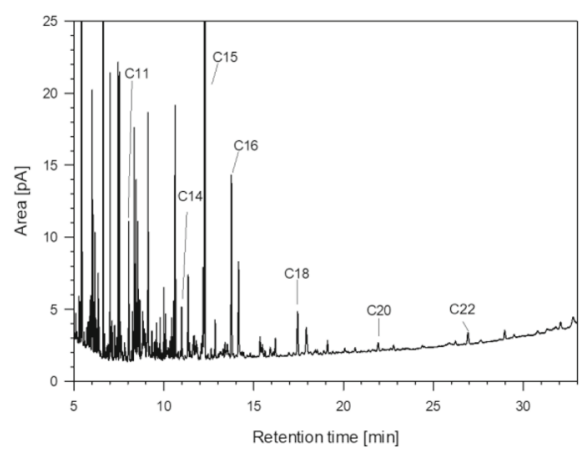

(iii)

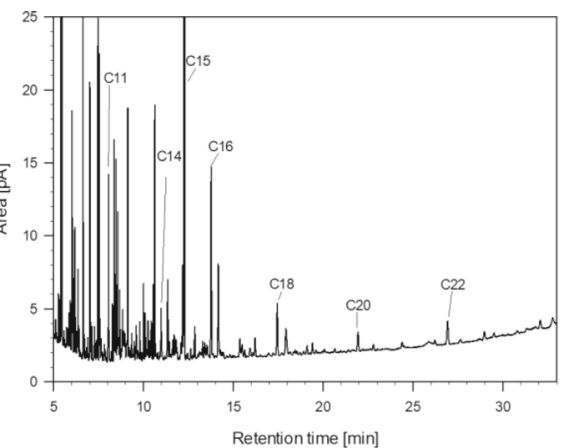

(ii)

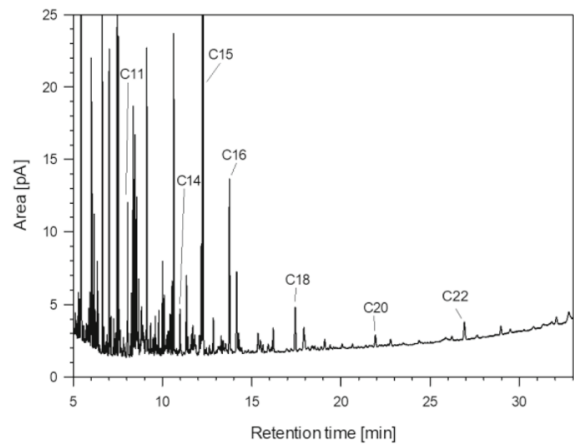

(iv)

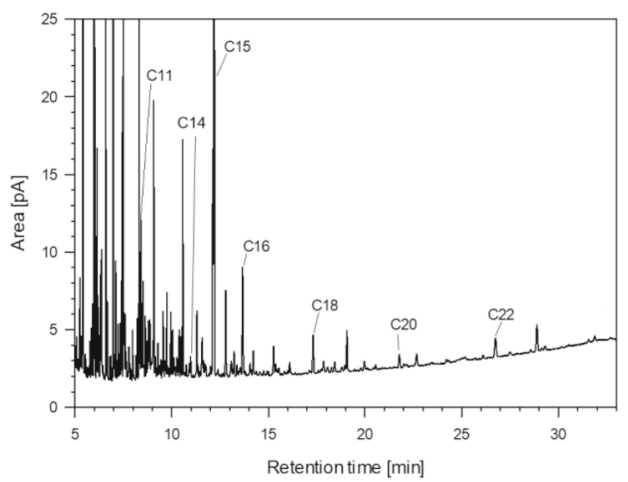

(v)

Fig. 8 Representative GC-FID chromatograms of fingerprint residue from donor E, i chloroform:ethanol (1:1), ii acetone:chloroform:hexane (1:1:1), iii hexane:isopropanol (3:2), iv chloroform:methanol (3:1), and $\mathbf{v}$ chloroform

recovery ratios of different treatment methods showed that the cotton swab method $(80.2 \%)$ was relatively more successful than other collection methods. There was variation in the recovery ratio among treatment methods. However, there was no significant difference between the four treatment methods. The next highest recovery ratio was obtained with pipette handing (77.7 \%), followed by rotator shaking (74.8\%), and tape lifting (67.6 \%) for C11 (Fig. 9). All treatment trials involved 10 repetitions. The total quantity of fatty acids (FA) from donors collected using different treatment methods showed high recovery (Fig. 10). Significant differences were observed between cotton swabbing and all other treatment methods. From the eight donors, eight fatty acids were obtained with total amounts of 4.6-26.6 $\mu \mathrm{g}$ per print. The detected saturated fatty acids were $\mathrm{C} 14, \mathrm{C} 16, \mathrm{C} 18, \mathrm{C} 20$, and $\mathrm{C} 22$, and unsaturated fatty acids were $\mathrm{C} 18: 1 \mathrm{n} 9 \mathrm{c}, \mathrm{C} 22: 1 \mathrm{n} 9$, and C22:6n3. Commonly detected fatty acids from all donors were C16 and C18. The quantity recovered from the treatment methods showed that cotton swab best extracted the remaining saturated fatty acids at statistically significant levels (Fig. 11). The result for unsaturated fatty acids showed large donor dependence. In addition, detected fatty acids also showed large dependence on treatment methods. 


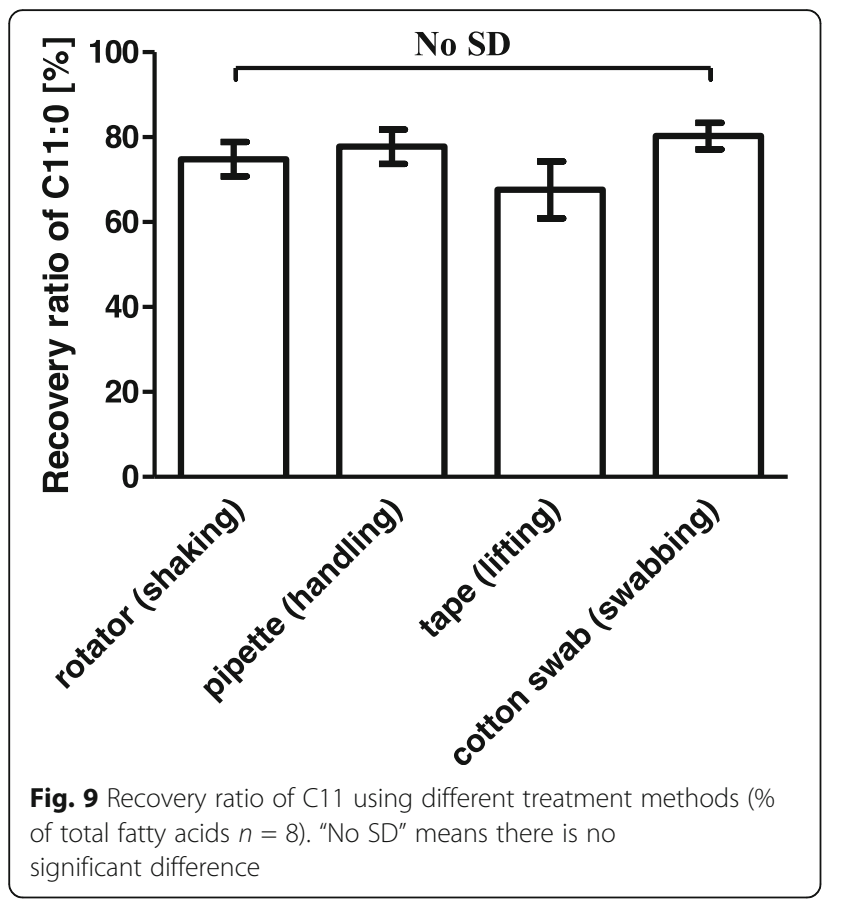

\section{Discussion}

In a review paper by Girod et al., 23 fatty acid components were identified in fingerprint residue, including even carbon numbers (C8 C24), odd carbon numbers (C9, C13 23), and unsaturated carbons (C14:1, C15:1, C16:1, C17:1, C18:1, C18:2) (Gird et al. 2012). The

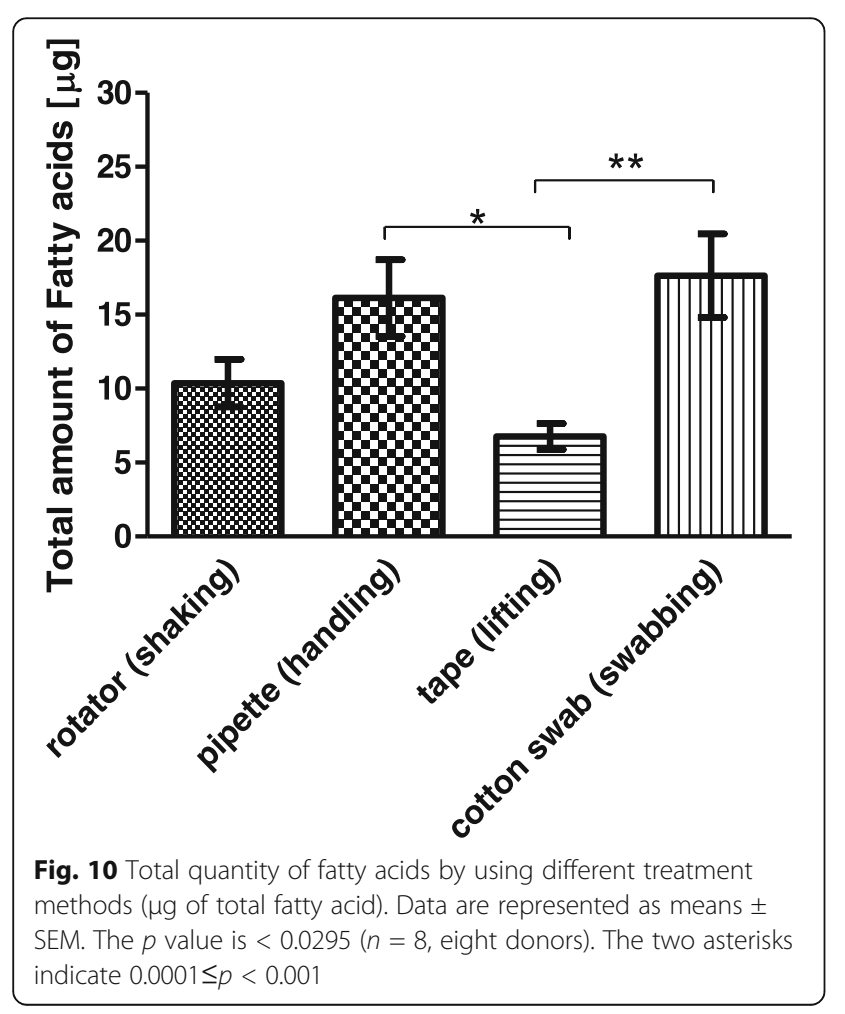

purpose of our research is to compare the difference of efficiency associated with the extraction solvents and treatment methods of fatty acids detected in groomed fingerprints. According to the results of the Croxton study, very small amounts of each fatty acid are detected in natural fingerprints (20 200 ng). In the authors' preliminary study, not shown, C12-C24 were not well detected in natural fingerprints. Considering the sensitivity of GC-FID, the minimum amounts of fatty acid are more than hundreds of nanograms. Thus, it is impossible to compare extraction efficiency of natural fingerprints. Therefore, even though fatty acids are overrepresented in them, we used groomed fingerprints. In this study, a high recovery ratio and various amounts of fatty acids were obtained from chloroform:ethanol (1:1) and cotton swab. These results showed that solvent solubility had an important effect on recovery ratio and amount of fatty acids. Solubility data showed that normal saturated fatty acids are generally more soluble in chloroform and less soluble than in any of the organic solvents investigated (Table 8) (http://cyberlipid.gerli.com/lipids/fattyacids/.). Fatty acids are carboxylic acids with long aliphatic chains that are easily extracted with non-polar solvents due to their non-polar characteristics. Methanol and ethanol have high polarity, and hexane is non-polar. According to Widjaja et al., hexane is not a suitable solvent to extract lipid. However, in a study on Botryccous, Chlorella vulgaris, and Scenedesmos, hexane, and iso-propanol mixtures showed better results at different temperatures and pressures (Widjaja et al. 2009). McNichol et al. showed that polar solvents like chloroform/methanol, ethanol, and acetone had higher lipid recoveries than nonpolar solvents like hexane (McNichol et al. 2012). In addition, Seth et al. showed that low recoveries and large loss can be due to evaporation of solvents like chloroform and dichloromethane. Thus, we suppose that the combination of chloroform and ethanol (1:1) can result in high recovery of fatty acids due to their complementary characteristics like solubility and high volatility.

Cotton swabbing and tape lifting methods are usually used to collect DNA samples. However, tape lifting did not remove all adhesive material. Rather, the recovery ratio using a pipette was higher than that of tape lifting. The pipette handling method was simpler than the other three methods and saved a significant amount of time. However, meticulous attention was required.

Wiping was necessary because solvent rinsing was not sufficient to remove material for acceptable instrumental analysis. It is possible that other substrates (e.g., paper) might allow for mere rinsing of the fingerprint from the substrate. However, wiping was necessary for glass. Additionally, glass materials provided the lowest background because impurities can easily be removed through a cleaning process, so glass surfaces were chosen for this work. 


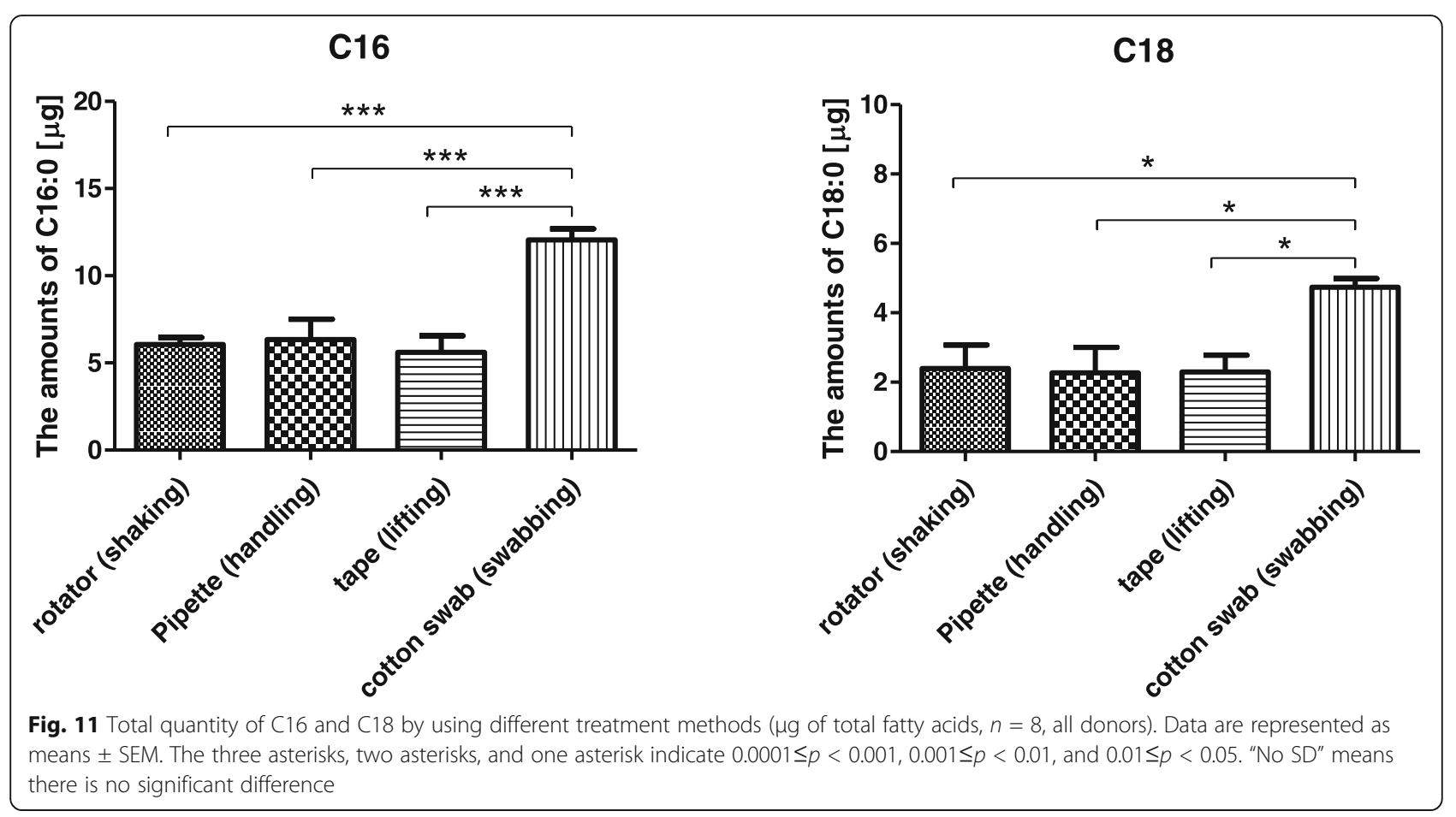

Fatty acids are a group of lipids that are most commonly analyzed by GC. This method is used for biological samples containing compounds with chain lengths in the range $\mathrm{C} 4$ to $\mathrm{C} 24$. GC analysis of fatty acids was performed following conversion to apolar methyl ester derivatives. The GC can be used to analyze fatty acids either as free fatty acids or as fatty acid methyl esters. Methyl esters are favorite derivatives for GC analysis of fatty acids. GC-FID with a methylation process allows polar compounds to be analyzed more readily, and the results can be quantified more generally, economically, and simply than the GCMS method (Young et al. 2012; Zhang et al. 2015). After fatty acid extraction, it is necessary to transform the constituents into specific derivatives to render them more volatile. In fingerprints, free fatty acids appear in the form of triglycerides composed of glycerol and three fatty acids. Thus, polar solvents and non-polar solvents can be effective in degrading non-polar fatty acids and polar glycerol (Emerson et al. 2011).

\section{Conclusions}

In this study, we tried to find stable and reproducible methods to quantify fatty acids in a fingerprint. The GCFID method was employed for direct quantitative analysis. The experimental result showed that the extraction efficiency of fatty acids in fingerprints differed significantly for different solvents and treatment methods. The most efficient extraction solvent and treatment methods for fatty acids detected in latent fingerprints were chloroform: ethanol $(1: 1, \mathrm{v} / \mathrm{v})$ and cotton swab, respectively. Therefore, in a non-porous substrate, solvent combinations with cotton swab methods will decrease extraction loss related to fatty acid compositions in fingerprints. Although we used groomed fingerprints, we found a reliable methodology. This method can be used to efficiently extract fatty acids from a latent fingerprint. Further work will involve the application of this method to analyze fatty acids detected in latent fingerprints based on external conditions such as temperature or age.

Table 8 Solubility data of fatty acids in organic solvents $[\mathrm{g} / \mathrm{L}]$

\begin{tabular}{lllllllll}
\hline Carbon number & Chloroform & Benzene & Cyclohexane & Acetone & Ethanol 95\% & Acetic acid & Methanol & Acetonitrile \\
\hline 10 & 3260 & 3980 & 3420 & 4070 & 4400 & 5670 & 5100 & 660 \\
12 & 830 & 936 & 680 & 605 & 912 & 818 & 1200 & 76 \\
14 & 325 & 292 & 215 & 159 & 189 & 102 & 173 & 18 \\
16 & 151 & 73 & 65 & 53.8 & 49.3 & 21.4 & 37 & 4 \\
18 & 60 & 24.6 & 24 & 15.4 & 11.3 & 1.2 & 1 & $<1$ \\
\hline
\end{tabular}




\section{Abbreviations}

CN: Chlorononane; FA: Fatty acids; FAME: Fatty acid methyl ester; GC-FID: Gas chromatography-flame ionization detection; GC-MS: Gas chromatography mass spectrometry; IFRG: International Fingerprint Research Group; MSTFA: $N$-methyl- $N$-(trimethylsilyl)-tri-fluoroacetamide

\section{Acknowledgements}

This research was supported by the Projects for Research and Development of Police science and Technology under Center for Research and Development of Police science and Technology and Korean National Police Agency (PAH000001); the National Research Foundation of Korea (NRF) grant funded by the Korea government (Ministry of Education) (NRF-2017R1D1A1B03030163) and (MSIP) (No. NRF-2018M3C1B7020722); the Korea Health Technology R\&D Project through the Korea Health Industry Development Institute (KHIDI), funded by the Ministry of Health \& Welfare, Republic of Korea (grant number:) (HI14C1277); and Basic Science Research Program through the National Research Foundation of Korea (NRF) funded by the Ministry of Science, ICT \& Future Planning (NRF-2017M3A9E9072939), and Grant 26-2015-0030 from the Seoul National University Hospital Research Fund. The authors would like to thank Ji Hyun Lee in Chromatography Laboratory, National Instrumentation Center for Environmental Management for his technical help and comments.

\section{Author's contributions}

This study was designed by YM, WS, and EJ. The experimental work was performed by $Y H, M W, X J, L X$, and $Y L$. JA and GH have contributed for the statistical analysis. YM and OK drafted the manuscript. BJ interpreted the data. JH, WK, and TH guided the research and modified the manuscript. All authors read and approved the final manuscript.

\section{Funding}

Center for Research and Development of Police science and Technology and Korean National Police Agency (PA-H000001)

The Korea government (Ministry of Education) (NRF-2017R1D1A1B03030163) The Korea government (Ministry of Science, ICT and Future Planning) (MSIP) (No. NRF-2018M3C1B7020722)

The Ministry of Health \& Welfare, Republic of Korea (Grant Number:) (HI14C1277)

Ministry of Science, ICT \& Future Planning (NRF-2017M3A9E9072939)

Seoul National University Hospital Research Fund (Grant 26-2015-0030)

\section{Availability of data and materials}

Data sharing is not applicable in this article as no datasets were generated or analyzed during the current study.

\section{Competing interests}

The authors declare that they have no competing interests.

\section{Author details}

${ }^{1}$ Interdisciplinary Program in Stem Cell Biology, College of Medicine, Seoul National University, Seoul, South Korea. ${ }^{2}$ Chromatography Laboratory, National Instrumentation Center for Environmental Management, Seoul National University, Seoul, South Korea. ${ }^{3}$ Interdisciplinary Program in Bioengineering, College of Engineering, Seoul National University, Seoul, South Korea. ${ }^{4}$ Department of Chemistry, College of Natural Sciences, Seoul National University, Seoul, South Korea. ${ }^{5}$ Department of Plastic and Reconstructive Surgery, Beijing Tongren Hospital, Capital Medical University, Beijing, People's Republic of China. ${ }^{6}$ Occupational Environmental Medicine, CHA, Gumi Medical Center, CHA University, Gumi, South Korea. ${ }^{7}$ Metabolic Regulation Research Center, Korea Research Institute of Bioscience and Biotechnolgy (KRIBB), Daejeon, South Korea. ${ }^{8}$ Department of Plastic and Reconstructive Surgery, Institute of Human-Environment Interface Biology, College of Medicine, Seoul National University, 101 Daehak-ro, Jongno-gu, Seoul, South Korea.

\section{Received: 24 April 2019 Accepted: 13 August 2019}

\section{Published online: 31 August 2019}

\section{References}

Antoine KM, Mortazavi S, Miller AD, Miller LM. Chemical differences are observed in children's versus adults' latent fingerprints as a function of time. J Forensic Sci. 2010;55:2.
Archer NE, Charles Y, Elliott JA, Jickells S. Changes in the lipid composition of latent fingerprint residue with time after deposition on a surface. Forensic Sci Int. 2005;154:224-39.

Asano KG, Bayne CK, Horsman KM, Buchanan MV. Chemical composition of fingerprints for gender determination. J Forensic Sci. 2002;47:4.

Bailey MJ, Randall EC, Costa C, et al. Analysis of urine, oral fluid and fingerprints by liquid extraction surface analysis coupled to high resolution MS and MS/MS opportunities for forensic and biomedical science. Anal Methods. 2016:8:3373.

Bobev K. Fingerprints and factors affecting their conditions. J Forensic Ident. 1995:45:176-83.

A.M. Bohanan, C, J. Forensic Ident. 48 (5) (1998) 570-573.

Cadd CJ, Mota L, Werkman D, Islam M, Zuidberg M, de Puit M. Extraction of fatty compounds from fingerprints for GCMS analysis. Anal Methods. 2015a;7:1123.

Cadd S, Islam M, Manson P, Bleay S. Fingerprint composition and aging: a literature review. Sci Justice. 2015b;55:219-38.

Croxton RS, Baron MG, Butler D, Kent T, Sears VG. Development of a GC-MS method for the simultaneous analysis of latent fingerprint components. Forensic Sci. 2006;51:1329-33.

Croxton RS, Baron MG, Butler D, Kent T, Sears VG. Variation in amino acid and lipid composition of latent fingerprints. Forensic Sci Int. 2010;199:93-102.

Dodds ED, McCoy MR, Rea LD, Kennish JM. Gas chromatographic quantification of fatty acid methyl esters: flame ionization detection vs. Electron impact mass spectrometry. Lipids. 2005;40(4):419-28.

Emerson B, Gidden J, Lay JO, Durham B. Laser desorption/ionization time-off light mass spectrometry of triacylglycerols and other components in fingermark samples. J Forensic Sci. 2011;56(2):381-9.

Garcés R, Mancha M. One-step lipid extraction and fatty acid methyl esters preparation from fresh plant tissues. Anal Biochemist. 1993;211:139-43.

Gird A, Ramotowski R, Weyermann C. Composition of fingermark residue: a qualitative and quantitative review. Forensic Sci Int. 2012;223:10-24.

Girod A, Weyermann C. Lipid composition of fingermark residue and donor classification using GC/MS. Forensic Sci Int. 2014;238:68-82.

Girod A, Spyratou A, Holmes D, Weyermann C. Aging of target lipid parameters in fingermark residue using GC/MS: effects of influence factors and perspectives for dating purposes. Sci Justice. 2016;56:165-80.

Godswill N-N, Frank N-EG, Edson M-YJ, Emmanuel Y, Martin BJ, Hermine N-B, et al. GC-FID method development and validation parameters for analysis of palm oil (Elaeis guineensis Jacq.) fatty acids composition. Res Plant Sci. 2014; 2(3):53-66.

Guidelines for the assessment of fingermark detection techniques, International Fingerprint Research Group (IFGR) 2014

http://cyberlipid.gerli.com/lipids/fatty-acids/.

Koenig A, Girod A, Weyermann C. Identification of wax esters in fingermark residues by GC/MS and their potential use as aging parameters. Ident. 2011; 61:652.

Laakso TS, Laakso I, Hiltunen R. Analysis of fatty acids by gas chromatography and its relevance to research on health and nutrition. Anal Chim Acta. 2002; 465:39-62.

McNichol J, MacDougall KM, Melanson JE, McGinn PJ. Suitability of soxhlet extraction to quantify microalgal fatty acids as determined by comparison with in situ transesterification. Lipids. 2012;47:195-207.

Michalski S, Shaler R, Dorman FL. The evaluation of fatty acid ratios in latent fingermarks by gas chromatography/mass spectrometry (GC/MS) analysis. J Forensic Sci. 2013a;58:S1.

Michalski S, Shaler R, Dorman FL. The evaluation of fatty acid ratio in latent fingermarks by gas chromatography/mass spectrometry (GC/NS) analysis. J. Forensic Sci. 2013b;58:20.

Mink T, Voorhaar A, Stoel R, de Puit M. Determination of efficacy of fingermark enhancement reagents; the use of propyl chlorofomate for the derivatization of fingerprint amino acids extracted from paper. Sci Justice. 2013;53:301-8.

Mong GM, Petersen CE, Clauss TRW. Advanced fingerprint analysis project final report - fingerprint constituents: Pacific Northwest National Laboratory; 1999.

Ramotowski, et al. Composition of latent print residue. In: Lee HC, Gaensslen RE, editors. Advances in fingerprint technology. 2nd ed. Boca Raton: CRC Press; 2001. p. 63-104.

Rohatgi R, Kapoor AK. Development of latent fingerprints on wet non-porous surfaces with SPR based in basic fuchsin dye. Egypt J Forensic Sci. 2016;6: 179-84.

Steadman SA, Hoofer SR, Geering SC, King S, Bennett MA. Recovery of DNA from latent fingerprint tape lifts archived against matte acetate. J Forensic Sci. 2015;60(3):777-82. 
Stewart ME, Steele WA, Downing DT. Changes in the relative amounts of endogenous and exogenous fatty acids in sebaceous lipids during early adolescence. J Invest Dermatol. 1989;92:371-8.

Weyermann C, Roux C, Champod C. Initial results on the composition of fingerprints and its evolution as a function of time by GC/MS analysis. J Forensic Sci. 2011;56:102-8

Widjaja A, Chien C-C, Ju Y-H. Study of increasing lipid production from fresh water microalgae chlorella vulgaris. J Taiwan Institute Chem Eng. 2009:40:13-20.

Young KE, Quinn SM, Trumble SJ. Comparing gas chromatographic techniques used in fatty acid profiling of northern fur seals and steller sea lions from Lovushki island complex, Russia. Int J Appl Sci Technol. 2012;2:9.

Zhang H, Wang Z, Liu O. Development and validation of a GC-FID method for quantitative analysis of oleic acid and related fatty acids. J Pharm Anal. 2015; 5:223-30.

\section{Publisher's Note}

Springer Nature remains neutral with regard to jurisdictional claims in published maps and institutional affiliations.

\section{Submit your manuscript to a SpringerOpen ${ }^{\circ}$ journal and benefit from:}

- Convenient online submission

- Rigorous peer review

- Open access: articles freely available online

- High visibility within the field

- Retaining the copyright to your article

Submit your next manuscript at $\boldsymbol{\nabla}$ springeropen.com 